\title{
RESOLUTION PROPERTIES IN REGULARIZED DYNAMIC MRI RECONSTRUCTION
}

\author{
Kimberly A. Khalsa \\ BME Dept., The University of Michigan \\ kkhalsa@umich.edu
}

\author{
Jeffrey A. Fessler \\ EECS Dept., The University of Michigan \\ fessler@umich.edu
}

\begin{abstract}
In dynamic MRI, one is constantly addressing the tradeoff between spatial and temporal resolution. Regularized reconstruction methods may offer benefits in terms of this tradeoff. However, selection of the regularization parameters is challenging. In this work we examine the spatial and temporal resolution of penalized-likelihood image reconstruction for dynamic MRI, and present an accelerated method for computing the local impulse response. This method may prove advantageous for regularization parameter selection.
\end{abstract}

Index Terms - MRI, regularization, resolution

\section{INTRODUCTION}

The fundamental challenge in dynamic Magnetic Resonance (MR) imaging is the tradeoff between spatial resolution and temporal resolution. Most traditional dynamic image acquisition methods and associated image reconstruction methods have been based on simple k-space operations. One acquires a temporal sequence of incomplete k-space sample sets, each of which may cover a different portion of k-space. In dynamic contrast enhanced MRI (DCE-MRI), e.g., breast cancer studies, one also collects a full k-space reference dataset before contrast injection, and possibly a second reference dataset after the contrast has reached equilibrium. Then one applies temporal interpolation or "data sharing" to form a sequence of "complete" $k$-space datasets from those partial datasets and the full k-space reference data. Finally one applies a traditional image reconstruction method (inverse FFT) to those imputed k-space datasets to form the dynamic image sequence. The Keyhole method exemplifies this type of approach [1].

Temporal interpolation in k-space is based on an implicit assumption that the object is varying smoothly over time. We are investigating image reconstruction methods for dynamic MRI that use explicit temporal models in object space. We use iterative methods for fitting those models to the measured $\mathrm{k}$-space data using regularized estimators without attempting to synthesize missing k-space data. The reconstruction algorithm is based on minimizing a cost function that consists of two types of terms: a data-fit term and regularization terms

This material is based upon work supported by a National Science Foundation Graduate Research Fellowship, and NIH grant P01 CA 87634. that account explicitly for temporal smoothness and spatial regularity. We refer to this approach as "Temporal Regularization Use in Image Reconstruction" (TRUIR). Similar formulations have been studied in electrocardiography [2-4] and recently applied to simulated cardiac MRI data [5].

A challenging aspect of any such formulation is choosing appropriate regularization terms, as well as determining the relative weights of these terms. Regularization parameter choice can significantly influence the quality of the reconstructed images.

This work aims to address the issue of regularization parameter selection by analyzing the resolution properties of the TRUIR method, which is a penalized-likelihood reconstructor. To do this, we examine the local impulse response. Similar analysis of penalized-likelihood reconstruction for (static) tomography was presented in [6].

Because only a partial $\mathrm{k}$-space dataset is collected for each time frame, and the sampled k-space locations generally vary from frame to frame, the TRUIR method is not locally shiftinvariant in time. That is, reconstruction of an object in the $m+1$ st time frame will not simply be a time-shifted version of the reconstruction of the same object in the $m$ th frame. This complicates the analysis of the local impulse response for dynamic imaging in MRI compared to previous such analyses for Positron Emission Tomography (PET) [7]. In particular, previous methods based solely on FFT calculations are not directly applicable. By building on this previous work, we present an accelerated method to determine the local impulse response of the TRUIR formulation for dynamic MR.

In the following section we derive an approximation to the local impulse response that leads to faster computation. Then we present some results indicating that spatial resolution does not rely solely on the spatial regularization parameter, $\beta$, nor does temporal resolution solely dependent on the temporal regularization parameter, $\alpha$.

\section{THEORY}

\subsection{Cost Function Formulation}

In dynamic MRI the measured data is a collection of $M$ scans $\boldsymbol{y}_{1}, \ldots, \boldsymbol{y}_{M}$, where $\boldsymbol{y}_{m}=\left(y_{m 1}, \ldots, y_{m, N_{m}}\right)$ and $N_{m}$ denotes the number of $\mathrm{k}$-space samples acquired for the $m \mathrm{th}$ 
scan. We parameterize the dynamic object $f\left(\vec{r}, t_{m}\right)$ during the $m$ th scan as:

$$
f\left(\vec{r}, t_{m}\right)=\sum_{j=1}^{n_{p}} \sum_{l=1}^{M} x_{l j} b_{s}\left(\vec{r}-\vec{r}_{j}\right) b_{t}\left(t_{m}-t_{l}\right),
$$

where $b_{s}(\vec{r})$ and $b_{t}(t)$ are spatial and temporal basis functions, respectively. The spatial coordinates $\vec{r}_{j}$ are spaced equally, but temporal sample times $t_{l}$ may be unequally spaced. Because each image is displayed using $n_{p}$ pixels, a natural choice for the spatial basis function is $b_{s}(\vec{r})=\operatorname{rect}(\vec{r})$. In this work, we also use $b_{t}(t)=\operatorname{rect}(t)$, although, as will be discussed further, this may not be the most appropriate choice given our object model. Nonetheless, it simplifies our analysis.

Based on the MR signal equation (and ignoring relaxation and field inhomogeneity), one can show that the measurement model becomes

$$
\begin{gathered}
\mathrm{E}\left[\boldsymbol{y}_{m}\right]=\mathbf{A}_{m} \boldsymbol{x}_{m} \\
{\left[\mathbf{A}_{m}\right]_{i j}=B\left(\vec{\nu}_{m i}\right) e^{-i 2 \pi \vec{\nu}_{m i} \cdot \vec{r}_{j}},}
\end{gathered}
$$

where $\boldsymbol{x}_{m}=\left(x_{m 1}, \ldots, x_{m, n_{p}}\right)$, and $B(\vec{\nu})$ is the Fourier Transform of $b(\vec{r})$ [8]. Note that because we are using a rect basis function in time, each $\boldsymbol{y}_{m}$ is a function of the object at a single time, $t_{m}$. Stacking the data, we can write

$$
\begin{gathered}
\mathrm{E}[\boldsymbol{y}]=\mathbf{A} \boldsymbol{x}, \\
\boldsymbol{y}=\left(\begin{array}{c}
\boldsymbol{y}_{1} \\
\vdots \\
\boldsymbol{y}_{M}
\end{array}\right), \quad \mathbf{A}=\left(\begin{array}{cccc}
\mathbf{A}_{1} & 0 & \ldots & 0 \\
0 & \mathbf{A}_{2} & 0 & \vdots \\
\vdots & 0 & \ddots & 0 \\
0 & \ldots & 0 & \mathbf{A}_{M}
\end{array}\right),
\end{gathered}
$$

with $\boldsymbol{x}$ stacked similarly to $\boldsymbol{y}$.

For complex Gaussian measurements, the negative loglikelihood is equivalent to

$$
L(\boldsymbol{x})=\frac{1}{2}\|\boldsymbol{y}-\mathbf{A} \boldsymbol{x}\|^{2} .
$$

Estimating $\boldsymbol{x}=\left(\boldsymbol{x}_{1}, \ldots, \boldsymbol{x}_{M}\right)$ by minimizing the negative log-likelihood would be equivalent to reconstructing each scan independently. However, because each $\boldsymbol{y}_{m}$ is undersampled in general, the ML estimator would be poorly conditioned and the reconstructions would have low spatial resolution. Instead, we propose the TRUIR cost function, which includes penalty terms that encourage spatial regularity and temporal smoothness:

$$
\begin{aligned}
\hat{\boldsymbol{x}} & =\underset{\boldsymbol{x}}{\operatorname{argmin}} \Psi(\boldsymbol{x}), \\
\Psi(\boldsymbol{x}) & =L(\boldsymbol{x})+\alpha R_{t}(\boldsymbol{x})+\beta R_{s}(\boldsymbol{x}) .
\end{aligned}
$$

We use a quadratic penalty in both space and time, with

$$
\begin{aligned}
& R_{t}(\boldsymbol{x})=\frac{1}{2}\left\|\mathbf{C}_{t} \boldsymbol{x}\right\|^{2} \\
& R_{s}(\boldsymbol{x})=\frac{1}{2}\left\|\mathbf{C}_{s} \boldsymbol{x}\right\|^{2}=\sum_{m=1}^{M} \frac{1}{2}\left\|\mathbf{C}_{s 0} \boldsymbol{x}_{m}\right\|^{2},
\end{aligned}
$$

where $\mathbf{C}_{s 0}$ is a spatial differencing matrix that compares neighboring pixels within an image and $\mathbf{C}_{s} \triangleq \mathbf{I} \otimes \mathbf{C}_{s 0}^{\prime} \mathbf{C}_{s 0}$. The temporal differencing matrix $\mathbf{C}_{t}$ compares pixels at the same spatial location in adjacent time frames. A first-order temporal penalty function looks like

$$
R_{t}(\boldsymbol{x})=\sum_{m=2}^{M} \frac{1}{2}\left\|\boldsymbol{x}_{m}-\boldsymbol{x}_{m-1}\right\|^{2},
$$

and higher order penalty functions may also be reasonable. Letting $R(\boldsymbol{x})=\alpha R_{t}(\boldsymbol{x})+\beta R_{s}(\boldsymbol{x})$, we can write

$$
\hat{\boldsymbol{x}}=\left[\mathbf{A}^{\prime} \mathbf{A}+\mathbf{R}\right]^{-1} \mathbf{A}^{\prime} \boldsymbol{y},
$$

where $\mathbf{R}$ is the Hessian of $R(\boldsymbol{x})$. This estimate can be computed using the conjugate gradient (CG) algorithm.

\subsection{Local Impulse Response}

For practical use of (4), one must understand how the regularization parameters $\alpha$ and $\beta$ in (3) affect the spatial and temporal resolution of the reconstructed image. One method of analyzing resolution is to look at the local impulse response [6]. Noting that $\mathrm{E}(\boldsymbol{y})=\mathbf{A} \boldsymbol{x}$, the local impulse response near the $j$ th pixel is

$$
\boldsymbol{l}_{j}=\left[\mathbf{A}^{\prime} \mathbf{A}+\mathbf{R}\right]^{-1} \mathbf{A}^{\prime} \mathbf{A} \boldsymbol{e}_{j} .
$$

This expression could be evaluated using the CG algorithm, but computation would be slow as the matrices are quite large and non-sparse.

We can simplify the expression for $\boldsymbol{l}_{j}$ by using the relationship between circulant matrices and FFTs. Namely, the eigenvalues of a circulant matrix are the DFT coefficients of the first column of the matrix [9]. Because the operators $\mathbf{A}_{m}^{\prime} \mathbf{A}_{m}$ and $\mathbf{C}_{s 0}^{\prime} \mathbf{C}_{s 0}$ are locally shift invariant in the spatial dimension (but not in the temporal dimension), they can be approximated by circulant matrices and have the following orthonormal eigenvector decompositions:

$$
\begin{aligned}
\mathbf{A}_{m}^{\prime} \mathbf{A}_{m} & \approx \mathbf{Q} \mathbf{\Lambda}_{m} \mathbf{Q}^{\prime} \\
\mathbf{C}_{s 0}^{\prime} \mathbf{C}_{s 0} & \approx \mathbf{Q} \boldsymbol{\Omega} \mathbf{Q}^{\prime},
\end{aligned}
$$

which implies

$$
\mathbf{A}^{\prime} \mathbf{A} \approx(\mathbf{I} \otimes \mathbf{Q}) \mathbf{\Lambda}(\mathbf{I} \otimes \mathbf{Q})^{\prime}
$$

where

$$
\boldsymbol{\Lambda}=\left(\begin{array}{cccc}
\boldsymbol{\Lambda}_{1} & 0 & \ldots & 0 \\
0 & \boldsymbol{\Lambda}_{2} & 0 & \vdots \\
\vdots & 0 & \ddots & 0 \\
0 & \ldots & 0 & \boldsymbol{\Lambda}_{M}
\end{array}\right)
$$

and $\mathbf{I}$ is the $M \times M$ identity matrix. Combining (5), (6), and (7), we get

$$
\begin{aligned}
\boldsymbol{l}_{j} & \approx(\mathbf{I} \otimes \mathbf{Q}) \hat{\boldsymbol{x}}_{f} \\
\hat{\boldsymbol{x}}_{f} & \triangleq\left[\boldsymbol{\Lambda}+\beta(\mathbf{I} \otimes \boldsymbol{\Omega})+\alpha \mathbf{C}_{t}^{\prime} \mathbf{C}_{t}\right]^{-1} \boldsymbol{\Lambda}\left(\mathbf{I} \otimes \mathbf{Q}^{\prime}\right) \boldsymbol{e}_{j} .
\end{aligned}
$$




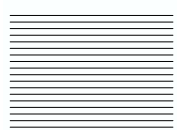

$t_{1}$

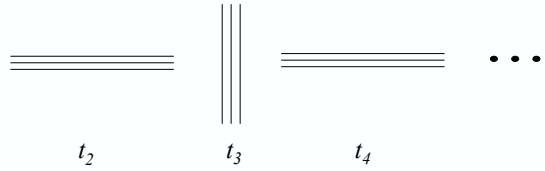

Fig. 1. K-space trajectory used in simulations.

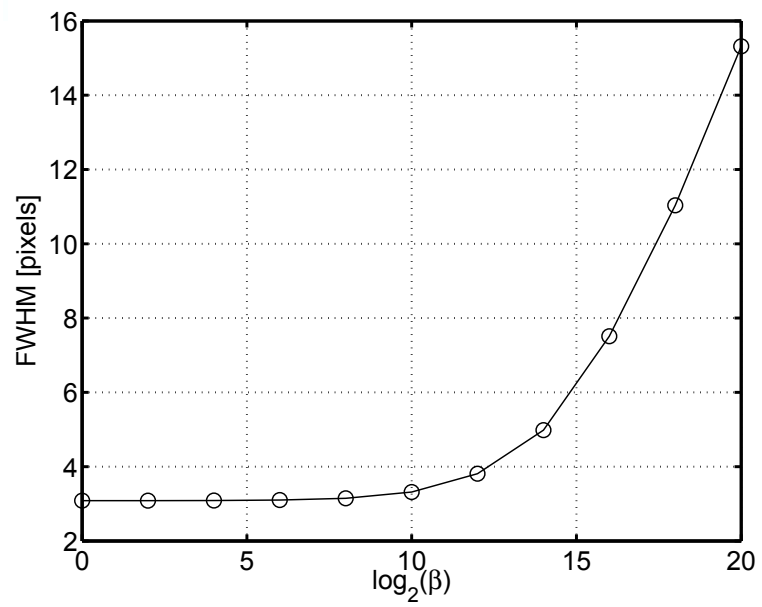

Fig. 2. Angularly averaged FWHM of spatial PSF for TRUIR as a function of spatial regularization parameter $\beta$.

While the inverse term in (9) is not diagonal, it composed of two diagonal matrices, $\boldsymbol{\Lambda}$ and $\beta(\mathbf{I} \otimes \boldsymbol{\Omega})$, and one sparse matrix $\alpha \mathbf{C}_{t}^{\prime} \mathbf{C}_{t}$. Thus it should be relatively quick to invert. Due to the approximations and simplifications described above, computing $\hat{\boldsymbol{x}}_{f}$ in (9) is much faster than computing $\boldsymbol{l}_{j}$ in (5). Once we have $\hat{\boldsymbol{x}}_{f}$, we obtain $\boldsymbol{l}_{j}$ using (8) through a simple inverse (spatial) FFT.

\section{RESULTS}

We simulated dynamic MR scans using the k-space trajectory shown in Fig. 1. We generated k-space data for one reference frame and eight subsequent frames, each undersampled by a factor of $1 / 16$. For the partially sampled frames, we alternated the phase encode and readout directions with each frame.

We computed the local impulse response using the accelerated method in (8) for many values of $\alpha$ and $\beta$. For this study, the accelerated method was over 3 times faster than evaluating the original expression for the local impulse response (5).

Fig. 2 shows the effect of the spatial regularization parameter $\beta$ on the Full Width at Half Maximum (FWHM) of the Point Spread Function (PSF) in space. The temporal regularization parameter $\alpha$ mainly affects the temporal PSF, and this dependence is shown in Fig. 3.

To gain a better understanding of how parameter choice

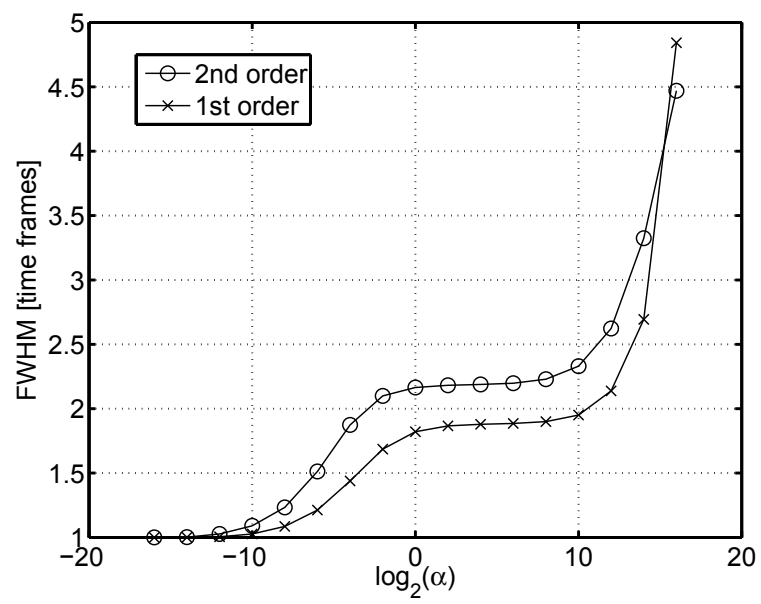

Fig. 3. FWHM of temporal PSF for TRUIR as a function of temporal regularization parameter $\alpha$.

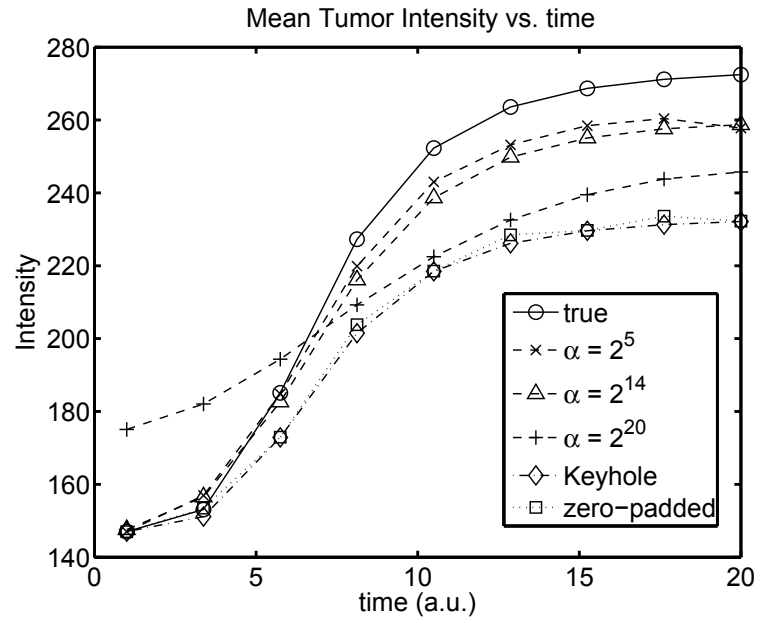

Fig. 4. Enhancent curve for simulated lesion and reconstructions

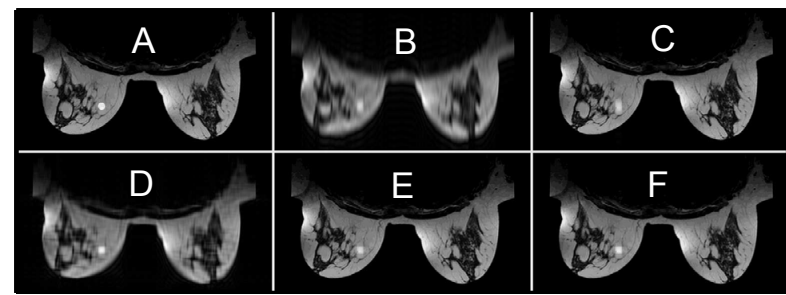

Fig. 5. True image (A) and reconstructions with zero-padding (B), Keyhole (C), TRUIR with $\alpha=2^{5}$ (D), TRUIR with $\alpha=$ $2^{20}(\mathrm{E})$, TRUIR with $\alpha=2^{14}(\mathrm{~F})$. 
affects dynamic images, we performed a simulation of contrast agent uptake using a real bilateral breast image with an inserted (simulated) circular lesion. The lesion exhibited enhancement over time according to the curve in Fig. 4, while the rest of the image remained static.

We reconstructed the data using $\beta=2^{4}$ and first order temporal regularization with several values of $\alpha$. Fig. 5 shows the reconstructed images from the 5 th time frame; zero-padded reconstruction and Keyhole reconstruction are shown for reference. The "tumor" enhancement curves from the reconstructed images appear in Fig. 4. Using an $\alpha$ that is too large degrades the temporal dynamics of the reconstructed sequence, as can be seen for $\alpha=2^{20}$ in Fig. 4. The reconstructed image from the middle frame of the dynamic sequence, shown in Fig. 5(E), has good spatial resolution.

In this case the large weighting of the temporal regularization term in the cost function (3) enforces strong correlation between the reference and partial datasets. The result is a sequence of reconstructed images with excellent spatial resolution, but flattened enhancement curve, i.e., poor temporal resolution.

Choosing $\alpha$ too small can also degrade the quality of the reconstructed images. Fig. 5(D) shows a reconstructed image using $\alpha=2^{5}$. This image appears quite blurry because the temporal regularization term in (3) is not large enough to overcome the spatial blur (due to undersampling of the kspace data in the individual frames) represented by the loglikelihood term. Note that the corresponding enhancement curve in Fig. 4 shows good temporal resolution.

We need $\alpha$ large enough to provide adequate "connectivity" between the frames (especially with the reference frame), but small enough so that the reconstructed image sequence correctly reflects dynamic changes in the object. In this simulation, $\alpha=2^{14}$ provided such a balance. The reconstructed enhancement curve in Fig. 4 is a good fit to the true enhancement curve, and the reconstructed image in Fig. 5(F) has good spatial resolution.

\section{DISCUSSION}

We have presented a fast method to compute the local impulse response for quadratic penalized least-squares reconstruction of a dynamic MRI sequence. Our results show that the local impulse response depends heavily on the choice of both temporal regularization parameter $\alpha$, and spatial regularization parameter $\beta$. This analysis is a first step in an attempt to provide guidelines for choosing these parameters.

Future work will also re-examine the choice of temporal basis function in parameterization of the dynamic object (1). Here we have used a rect function, but an underlying theme of our problem formulation is temporal smoothness, thus this choice may be inappropriate. Using B-splines as temporal basis functions may prove more reasonable, and we are currently investigating their use in this problem.

\section{REFERENCES}

[1] J. J. Van Vaals, M. E. Brummer, W. T. Dixon, H. H. Tuithof, H. Engels, R. C. Nelson, B. M. Gerety, J. L. Chezmar, and J. A. Den Boer, "Keyhole method of accelerating imaging of contrast agent uptake," J. Mag. Res. Im., vol. 3, no. 4, pp. 671-5, July 1993.

[2] H. S. Oster and Y. Rudy, "The use of temporal information in the regularization of the inverse problem of electrocardiography," IEEE Trans. Biomed. Engin., vol. 39, no. 1, pp. 65-75, Jan. 1992.

[3] D. H. Brooks, G. F. Ahmad, R. S. MacLeod, and G. M. Maratos, "Inverse electrocardiography by simultaneous imposition of multiple constraints," IEEE Trans. Biomed. Engin., vol. 46, no. 1, pp. 3-18, Jan. 1999.

[4] F. Greensite, "The temporal prior in bioelectromagnetic source imaging problems," IEEE Trans. Biomed. Engin., vol. 50, no. 10, pp. 1152-9, Oct. 2003.

[5] A. Samsonov, "Spatial-temporal regularization framework for dynamic MRI series reconstruction," in Proc. Intl. Soc. Mag. Res. Med., 2005, p. 2309.

[6] J. A. Fessler and W. L. Rogers, "Spatial resolution properties of penalized-likelihood image reconstruction methods: Spaceinvariant tomographs," IEEE Trans. Im. Proc., vol. 5, no. 9, pp. 1346-58, Sept. 1996.

[7] E. Asma and R. M. Leahy, "Mean and covariance properties of dynamic PET reconstructions from list-mode data," IEEE Trans. Med. Imag., vol. 25, no. 1, pp. 42-54, Jan. 2006.

[8] B. P. Sutton, D. C. Noll, and J. A. Fessler, "Fast, iterative image reconstruction for MRI in the presence of field inhomogeneities," IEEE Trans. Med. Imag., vol. 22, no. 2, pp. 178-88, Feb. 2003

[9] J. A. Fessler, Image reconstruction: Algorithms and analysis, ?, 2006, Book in preparation. 